\title{
PICO-WATTS RANGE UNCOOLED INFRARED DETECTOR BASED ON A FREESTANDING PIEZOELECTRIC RESONANT MICROPLATE WITH NANOSCALE METAL ANCHORS
}

\author{
Y. Hui, Z. Qian, G. Hummel, and M. Rinaldi \\ Northeastern University, Boston, Massachusetts, USA
}

\begin{abstract}
This paper reports on the first demonstration of an ultra-high resolution $\left(\sim 371 \mathrm{pW} / \mathrm{Hz}^{1 / 2}\right)$ uncooled infrared (IR) detector based on a high frequency $(136 \mathrm{MHz})$ Aluminum Nitride (AlN) piezoelectric resonant micro-plate completely released from the substrate and supported by two nanoscale Platinum $(\mathrm{Pt})$ anchors. For the first time, fully metallic tethers were employed to support the freestanding vibrating body of a piezoelectric resonator and provide electrical connection to it (the device anchors are conventionally defined in the piezoelectric layer). Such innovative design, with minimum anchor cross section, enabled the implementation of an uncooled resonant thermal detector with ultra-high thermal resistance $\left(\sim 10^{5} \mathrm{~K} / \mathrm{W}\right)$ and electromechanical performance (mechanical quality factor, $Q_{M} \approx 3133$ in air, and electromechanical coupling coefficient, $k_{t}{ }^{2} \approx 1.86 \%$ ). Such unique combination of high sensitivity $(\sim 2.1 \mathrm{~Hz} / \mathrm{nW})$, low noise performance $\left(\sim 0.78 \mathrm{~Hz} / \mathrm{Hz}^{1 / 2}\right)$ and high resonator figure of merit $\left(F O M=k_{t}{ }^{2} \cdot Q \approx 58.3\right)$ resulted in the first complete and compelling prototype of a low power $(\sim 11 \mathrm{~mW})$ and high performance MEMSCMOS resonant uncooled IR detector with detection limit pushed in $\sim 100 \mathrm{~s} \mathrm{pW} / \mathrm{Hz}^{1 / 2}$ range.
\end{abstract}

\section{INTRODUCTION}

The interest in uncooled IR detectors based on Micro/NanoElectro-Mechanical Systems (MEMS/NEMS) resonator technologies is steadily growing due to their potentially ultra-high resolution and unique advantages in terms of size and cost, compared to conventional cryogenically cooled semiconductor photon detectors [1-5]. The most important parameters that ought to be considered for the design and optimization of uncooled and miniaturized thermal detectors are the device sensitivity to absorbed radiation, the noise performance and the ease of readout (especially crucial for focal plane array implementations). All these three fundamental challenges are addressed in this work with the experimental demonstration of a novel micromechanical resonant structure that, by taking advantage of advanced material properties and innovative device engineering, is characterized by a unique set of application enabling features, such as: (a) high sensitivity, due to excellent isolation from the heat sink (enabled by the large thermal resistance associated with the nanoscale metal anchors); (b) ultra-low noise performance, due to the intrinsic high $Q$ of the resonant device (enabled by the improved confinement of acoustic energy in the resonant body of the device by using nanoscale metal anchors); (c) ease of readout, due to the excellent piezoelectric transduction properties of AIN at micro and nano scale which enables the use of a low power and self-sustained CMOS oscillator as direct frequency readout.

\section{DESIGN AND FABRICATION}

A high performance resonant IR detector is composed of a high quality factor, $Q$, MEMS resonator whose resonance frequency is highly sensitive to IR radiation [4]. When IR radiation is absorbed by the resonant structure, the temperature of the device increases (because of the large thermal resistance of the structure) resulting in a shift in resonance frequency due to the temperature coefficient of frequency (TCF) of the piezoelectric resonator [3]. The temperature rise of the resonator due to the incident IR power is given by:

$$
\Delta T=\frac{\eta Q_{p}}{\sqrt{G_{t h}^{2}+\omega^{2} C_{t h}^{2}}} \approx \eta Q_{p} R_{t h}
$$

where $\eta$ is the absorption coefficient of the resonator, $Q_{p}$ is the incident IR radiation power, $G_{t h}$ is the thermal conductance, $C_{t h}$ is the thermal capacity, and $\omega$ is the angular frequency of the incident IR radiation. When the incident IR radiation is constant, or slowly changing over time $(\omega \approx 0)$, the temperature rise of the resonator is directly proportional to the thermal resistance, $R_{t h}$, between the resonant body and the heat sink. Therefore, the implementation of a resonant structure extremely well isolated from the heat sink is crucial for the achievement of high temperature rise factor, thus high responsivity of the IR detector. The thermal isolation of a resonant thermal detector is mainly determined by the thermal resistance associated with the tethers connecting the freestanding vibrating body of the device to the substrate. In the case of a piezoelectric MEMS resonator, such anchors are conventional composed of a relatively thick piezoelectric layer (directly patterned in the same device layer forming the vibrating body of the resonator) and a relatively thin metal layer employed to route the electrical signal to the electrode placed on top or bottom (depending on the device design) of the freestanding piezoelectric body of the resonator [6]. By completely removing the relatively thick piezoelectric material from the anchors, hence minimizing their thicknesses (ultimately limited by the need of a thin metal layer for electrical routing), maximum thermal isolation of the device resonant body from the heat sink would be readily achieved. Furthermore, the increased acoustic impedance associated with such metal tethers with minimum cross section would act to reduce the energy loss through the device anchors (the acoustic energy would be well confined in the resonant body of the device) which has been identified as a significant source of quality factor, $Q$, degradation in piezoelectric MEMS resonators operating below $1 \mathrm{GHz}$ [7].

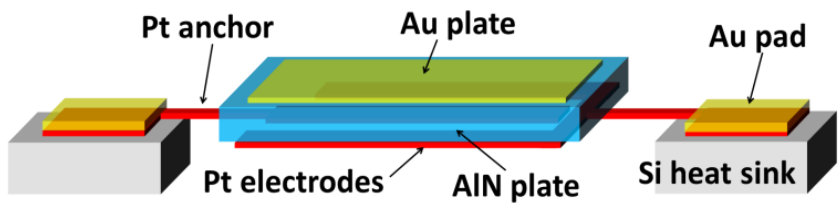

Figure 1: 3-dimensional representation of the AlN micro-plate resonator with nanoscale metallic (Pt) anchors.

According to these considerations, a new device concept, based on the use of fully metallic nanoscale tethers to support the resonant body of a piezoelectric MEMS resonator, is introduced in this work as an innovative design solution to maximize the thermal resistance and the electromechanical performance of piezoelectric MEMS resonant thermal detectors. The core of the proposed piezoelectric resonant uncooled thermal detector is an $\mathrm{AlN}$ 
piezoelectric resonant micro-plate (working at a higher order contour-extensional mode of vibration employing a lateral field excitation scheme [6]) completely released from the substrate and supported by two nanoscale Platinum $(\mathrm{Pt})$ anchors. Figure 1 shows the 3-dimensional representation of the proposed AlN micro-plate resonator with fully metallic nanoscale anchors: the freestanding vibrating body consists of a $450 \mathrm{~nm}$ thick AlN piezoelectric layer sandwiched between a $100 \mathrm{~nm}$ thick gold (Au) film as top electrically floating electrode and a $100 \mathrm{~nm}$ thick Platinum $(\mathrm{Pt})$ bottom inter-digital transducer (IDT); two nanoscale Pt tethers (100 $\mathrm{nm}$ thick, 3 6 $\mu \mathrm{m}$ wide and $22 \mu \mathrm{m}$ long) are employed to support the piezoelectric resonant body and provide electrical connection to it.

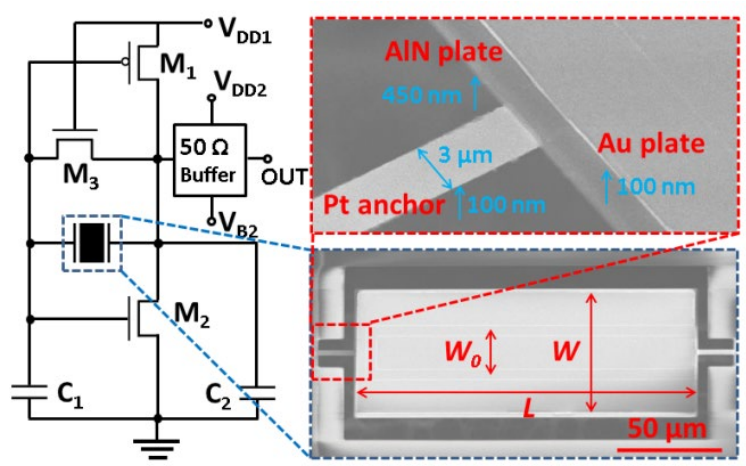

Figure 2: Schematic illustration of the CMOS amplifier and Scanning Electron Microscopy (SEM) images of the fabricated AlN resonator with nanoscle metal anchors: $L=200 \mu \mathrm{m}, W=75 \mu \mathrm{m}$ and $W_{0}=25 \mu \mathrm{m}$.

Such piezoelectric resonant micro-plate with nanoscale metal anchors is directly connected in the feedback loop of a selfsustaining CMOS amplifier as direct frequency readout [8]. The schematic illustration of the CMOS amplifier is shown in Figure 2: the circuit consists of a Pierce oscillator implemented by a CMOS inverting amplifier and a $50 \Omega$ buffer stage. The design of the CMOS circuit is fully described in $[9,10]$. The AlN resonant uncooled thermal detector was fabricated using a 4-mask postCMOS compatible microfabrication process [11], while the CMOS circuit was taped out in the ON Semiconductor $0.5 \mu \mathrm{m}$ CMOS process. The fabricated AlN resonator was directly wire-bonded to the CMOS chip, forming the first complete and compelling prototype of a high performance MEMS-CMOS resonant thermal detector.

\section{EXPERIMENTAL RESULTS}

The effectiveness of the proposed design solution was verified with the fabrication and characterization of $17 \mathrm{AlN}$ resonators employing different anchor configurations and operating at two different frequencies as listed in the table in Figure 3. The electrical response of each device was measured by an Agilent E5071C network analyzer after performing an open-short-load calibration on a standard substrate and the mechanical quality factor was extracted by modified Butterworth-Van Dyke (MBVD) model fitting (Figure 3-a). The thermal resistance, $R_{t h}$, of each resonator was estimated by 3-dimensional Finite Element Method (FEM) simulation using CMOSOL Multiphysics (Figure 3-b). The details of the 3D COMSOL simulations are described in [12]. The experimental characterization and the FEM analysis of the 17 fabricated devices indicate that both the mechanical quality factor and the thermal resistance of the AIN resonator were simultaneously improved by a large extent when fully metallic nanoscale Pt anchors were employed: $R_{t h}$ was improved by one order of magnitude (from $\sim 10^{4}$ to $\sim 10^{5} \mathrm{k} / \mathrm{W}$ ) and an average $Q_{M}$ improvement of $\sim 92 \%$ at $136 \mathrm{MHz}$ and $\sim 28 \%$ at $222 \mathrm{MHz}$ was recorded. Such simultaneous improvement in both mechanical quality factor and thermal resistance is crucial for the implementation of ultra-sensitive and low-noise MEMS resonant IR detectors.
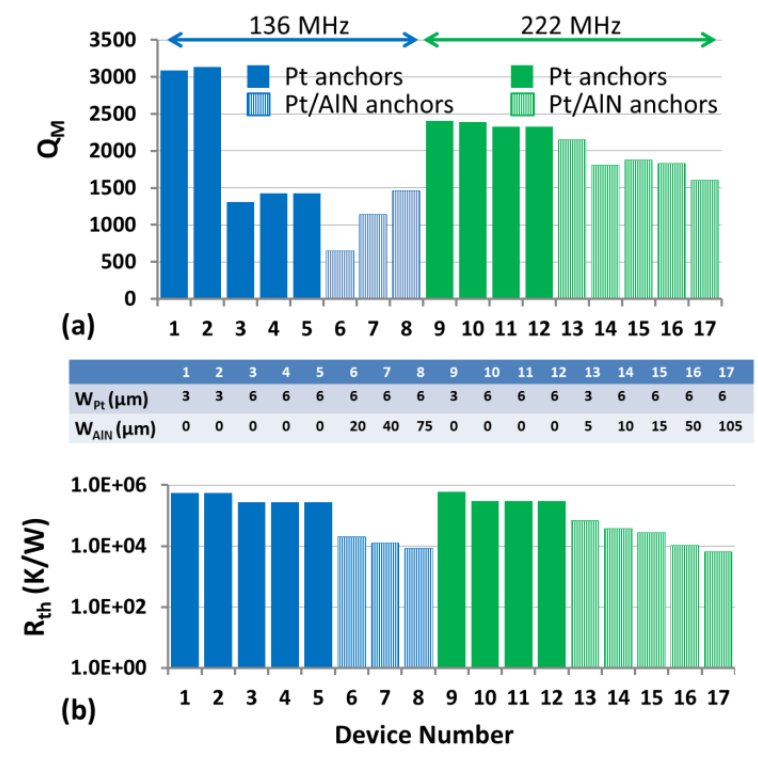

Figure 3: (a) Experimentally extracted mechanical quality factor values of 17 resonators employing different anchor configurations and operating at two different frequencies: solid bars represent the $Q_{M}$ of devices employing fully metallic Pt anchors while shaded bars represent the $Q_{M}$ of devices with conventional Pt/AlN anchors. The anchor width for each device is listed in the table (the anchor length and thickness are $22 \mu \mathrm{m}$ and $100 \mathrm{~nm}$ for all the resonators). (b) Finite Element Method (FEM) simulated thermal resistance, $R_{\text {th }}$, of the same 17 resonators.

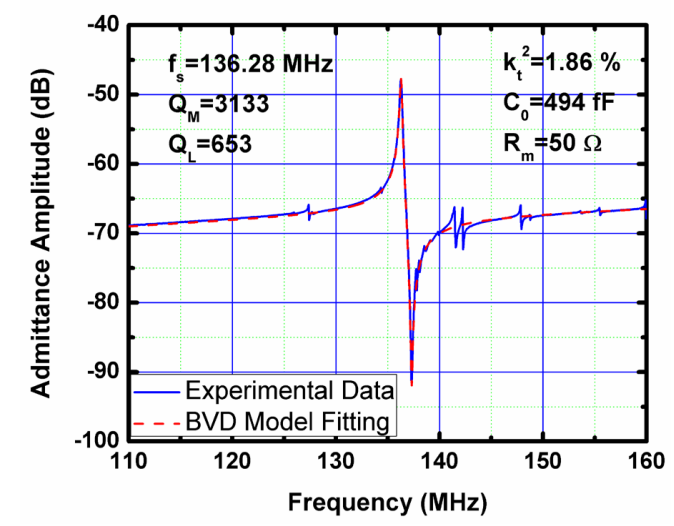

Figure 4: Measured admittance curve versus frequency and modified Butterworth-Van Dyke (MBVD) model fitting of the fabricated AlN resonator with nanoscale metal anchors. The relatively low value of loaded quality factor, $Q_{L}$, is due to the large electrical resistivity of the deposited Pt thin-film $(\sim 5 X$ the bulk value). Values of $Q_{L}$ comparable to $Q_{M}$ could be achieved by optimizing Pt deposition or exploring the use of different metals.

Maximum $Q_{M}$ and $R_{t h}$ were achieved for the $136 \mathrm{MHz}$ design employing $3 \mu \mathrm{m}$ wide $\mathrm{Pt}$ anchors. Therefore, this device 
configuration was employed for the demonstration of a complete prototype of MEMS-CMOS IR detector. The mechanical quality factor $Q_{M}$ and electromechanical coupling coefficient $k_{t}{ }^{2}$ were extracted by MBVD fitting and found to be $\sim 3133$ and $\sim 1.86 \%$, respectively (Figure 4). Such high electromechanical performance (Figure of Merit $F O M=k_{t}{ }^{2} \cdot Q_{M} \approx 58$ ) enabled the use of a compact and power efficient CMOS oscillator as direct frequency readout. The thermal resistance of the device was estimated by 3D FEM simulation and found to be $\sim 5.5 \times 10^{5} \mathrm{~K} / W$ (Figure 5-a), which is one order of magnitude higher than what typically achieved with conventional AIN resonator with AIN/Pt anchors [4]. The temperature distribution across the AlN resonant body was also simulated by applying an input power of $500 n \mathrm{~W}$ to the top $\mathrm{Au}$ plate of the resonator (simulating IR absorbed power). A maximum temperature rise of $\sim 274.3 \mathrm{mK}$ at the center of the resonant microplate and a maximum temperature difference of $\sim 5.5 \mathrm{mK}$ across the length of the resonator were recorded, translating into a relative temperature variation of only $\sim 2 \%$ (Figure 5-b). The achievement of such uniform temperature distribution across the AlN resonant body was enabled by the large thermal resistance of the anchors and the relatively low thermal resistance of the AlN micro-plate. The temperature coefficient of frequency (TCF) of the AlN resonator with nanoscale $\mathrm{Pt}$ anchors was measured using a temperature controlled RF probe station and a TCF of $-27.8 \mathrm{ppm} / \mathrm{K}$ was recorded. The frequency sensitivity of the AlN resonant device to absorbed radiation was calculated by multiplying the measured frequency sensitivity to temperature $(\sim-3788 \mathrm{~Hz} / \mathrm{K})$ by the simulated $R_{t h}\left(\sim 5.5 \times 10^{5} \mathrm{~K} / \mathrm{W}\right)$, resulting in an absolute responsivity of $\sim 2.1 \mathrm{~Hz} / \mathrm{nW}$, which is one order of magnitude higher than the one achieved with conventional resonators employing AlN/Pt anchors [8].
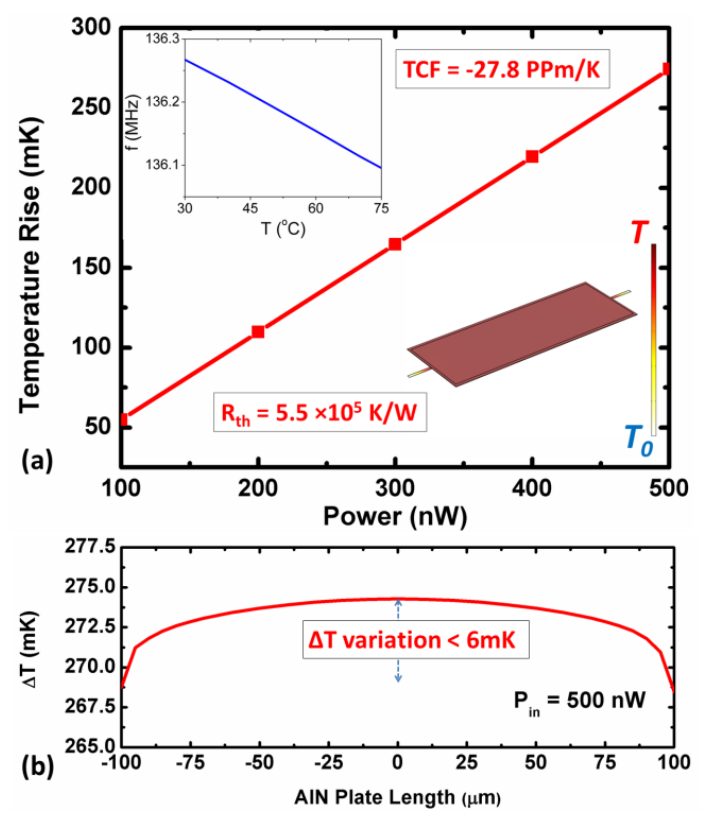

Figure 5: (a) 3-dimensional FEM simulated thermal resistance $\left(R_{t h}\right)$ of the MEMS resonant IR detector. The inset shows the measured TCF of $-27.8 \mathrm{ppm} / \mathrm{K}$. A device responsivity of $2.1 \mathrm{~Hz} / \mathrm{nW}$ was calculated by multiplying the measured TCF and simulated $R_{t h}$. (b) Simulated temperature distribution across the AlN resonant micro-plate. Extremely uniform heating of the device resonant body was achieved thanks to the large thermal resistance of the anchors and the relatively low thermal resistance of the AlN micro-plate.
The resonator was directly wire-bonded to the CMOS chip, forming a self-sustained piezoelectric MEMS-CMOS oscillator. In order to characterize the limit of detection of the sensor, the short term frequency stability of the oscillator output signal was characterized by measuring its Allan deviation [13]. The frequency measurements were taken with an Agilent 53230A frequency counter (Figure 6). A minimum Allan deviation of $\sim 4.5 \mathrm{~Hz}$ was recorded for a measurement time of $30 \mathrm{~ms}$, translating into a noiseinduced frequency fluctuation of $\sim 33 \mathrm{ppb}$ and a noise spectral density of $\sim 0.78 \mathrm{~Hz} / \mathrm{Hz}^{1 / 2}$. The Noise Equivalent Power (NEP) of the IR detector was extracted by dividing the measured noise spectral density $\left(0.78 \mathrm{~Hz} / \mathrm{Hz}^{1 / 2}\right)$ by the absolute value of the responsivity $(2.1 \mathrm{~Hz} / \mathrm{nW})$, and found to be $\sim 371 \mathrm{pW} / \mathrm{Hz}^{1 / 2}$. Such NEP is one order of magnitude lower than the one achieved with uncooled IR detector based on the same AIN technology but employing conventional AlN/Pt anchors [8], and 2 4X better than commercially available pyroelectric detectors [14].

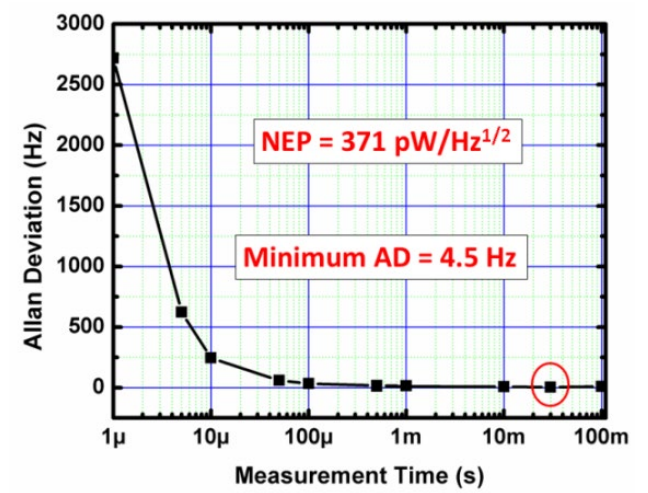

Figure 6: Measured Allan Deviation (AD) of the MEMS-CMOS oscillator: a minimum Allan Deviation of $\sim 4.5 \mathrm{~Hz}$ was recorded for a measurement time of $30 \mathrm{~ms}$.

The frequency response of the device prototype to IR radiation was characterized using a Bruker Vertex 70 FTIR and Hyperion microscope, shown in Figure 7. A broadband IR radiation from a Tungsten Halogen lamp $(0.25 \mu \mathrm{m}-100 \mu \mathrm{m})$ was optically filtered $(0.33 \mu \mathrm{m}-2.8 \mu \mathrm{m}$ transmission $)$ and chopped $(6.25 \mathrm{~Hz})$ before it was coupled into a Hyperion microscope used to focus the light onto the resonant body of the device. The frequency response of the MEMS-CMOS oscillator was monitored using an Agilent 53230A frequency counter. The measurement was performed in air.

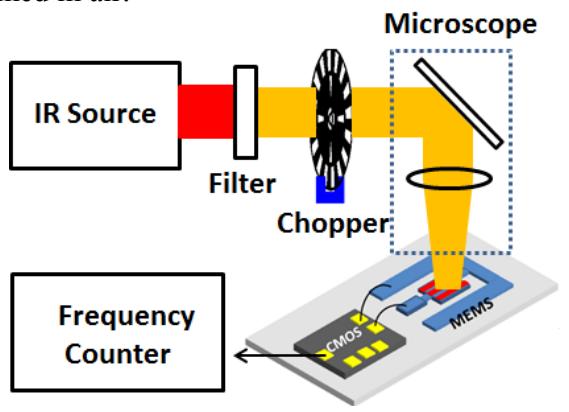

Figure 7: Schematic of the measurement set up used to characterize the response of the IR detector. Note that the measurement was performed without the use of a lock-in amplifier.

The measured output frequency response of the AlN piezoelectric MEMS-CMOS oscillator to near infrared (NIR) 
radiation is shown in Figure 8. Despite the fact that no specific IR absorbing element was integrated with the AlN resonator, an absolute frequency shift of $\sim 500 \mathrm{~Hz}$ was recorded when the device was exposed to NIR radiation. Such IR radiation induced frequency shift translates into an absorbed NIR power of $\sim 238 \mathrm{nW}$ (minimal IR absorption due to the intrinsic material loss), considering the device absolute responsivity of $2.1 \mathrm{~Hz} / \mathrm{nW}$. A thermal time constant of $\sim 20 \mathrm{~ms}$ was also experimentally recorded.

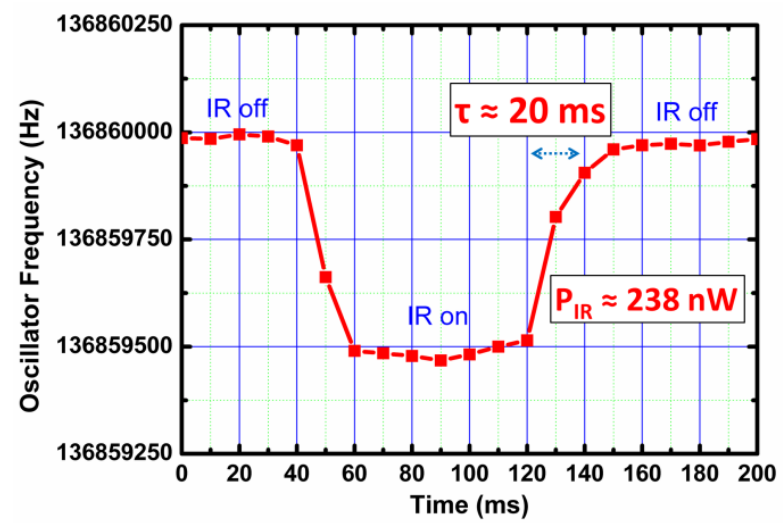

Figure 8: Measured frequency response of the IR detector to NIR radiation. Although no $I R$ absorbing material was integrated on the resonator, an output frequency shift of $500 \mathrm{~Hz}$ was recorded; corresponding to an absorbed IR power of $238 \mathrm{nW}$ (due to the intrinsic material losses). A thermal time constant of $\sim 20 \mathrm{~ms}$ was also recorded.

\section{CONCLUSION}

This paper demonstrates a new device concept, based on the use of fully metallic nanoscale tethers to support the resonant body of a piezoelectric MEMS resonator, which enables the implementation of piezoelectric MEMS resonators with maximum thermal resistance and quality factor. Such innovative design, with minimum anchor cross section, enabled the implementation of an uncooled resonant thermal detector with unprecedented performance: (1) the thermal resistance of the device was maximized $\left(R_{t h} \sim 5.5 \times 10^{5} \mathrm{~K} / \mathrm{W}\right.$, more than $10 \mathrm{X}$ improvement compared to conventional piezoelectric resonators) resulting in a very high frequency sensitivity to absorbed power $(\sim 2.1 \mathrm{~Hz} / \mathrm{nW})$. (2) The acoustic energy was effectively confined in the freestanding resonant body of the device resulting in excellent electromechanical performance (mechanical quality factor $Q_{M} \sim$ 3133 and electromechanical coupling coefficient $k_{t}{ }^{2} \sim 1.86 \%$ ) and ultra-low frequency noise $\left(\sim 0.78 \mathrm{~Hz} / \mathrm{Hz}^{1 / 2}\right)$. Thanks to such unique combination of high sensitivity and low noise performance, an ultra-low detection limit of $\sim 371 \mathrm{pW} / \mathrm{Hz}^{1 / 2}$ was achieved $(2 \sim 4 X$ better than commercially available pyroelectric detectors). Furthermore, by taking advantage of the high resonator figure of merit $\left(F O M=k_{t}^{2} \cdot Q \approx 58.3\right)$ a low power $(\sim 11 \mathrm{~mW})$ self-sustained CMOS oscillator was employed as direct frequency readout resulting in the first complete and compelling prototype of a high performance MEMS-CMOS resonant thermal detector with detection limit pushed in $\sim 100 \mathrm{~s} \mathrm{pW} / \mathrm{Hz}^{1 / 2}$ range.

\section{ACKNOWLEDGEMENT}

The authors wish to thank the staff of the George J. Kostas Nanotechnology and Manufacturing Facility, Northeastern University for their support in device fabrication. This project was supported by DARPA Young Faculty Award N66001-12-1-4221.

\section{REFERENCES}

[1] V. J. Gokhale, Y. Sui, and M. Rais-Zadeh, "Novel Uncooled Detector Based on Gallium Nitride Micromechanical Resonators", in proc. SPIE, vol. 8353, pp. 835319, 2012.

[2] M. B. Pisani, K. Ren, P. Kao, and S. Tadigadapa, "Application of Micromachined Y-Cut-Quartz Bulk Acoustic Wave Resonator for Infrared Sensing”, Journal of Microelectromechanical Systems, vol. 20, pp. 288-296, 2011.

[3] Y. Hui, and M. Rinaldi, "Fast and High Resolution Thermal Detector based on an Aluminum Nitride Piezoelectric Microelectromechanical Resonator with an Integrated Suspended Heat Absorbing Element", Applied Physics Letters, vol. 102, pp. 093501, 2013.

[4] Y. Hui, and M. Rinaldi, "High Performance NEMS Resonant Infrared Detector Based on an Aluminum Nitride Nano Plate Resonator", in proc. 17th International Conference on SolidState Sensors, Actuators and Microsystems (Transducers 2013), Barcelona, Spain, June 16-20, pp. 968-971, 2013.

[5] X. C. Zhang, E. B. Myers, J. E. Sader, and M. L. Roukes, "Nanomechanical Torsional Resonators for Frequency-Shift Infrared Thermal Sensing", Nano Letters, vol. 13, pp. 15281534, 2013.

[6] M. Rinaldi, and G. Piazza, "Effects of volume and frequency scaling in AlN contour mode NEMS resonators on oscillator phase noise", in proc. IEEE International Frequency Control Symposium, San Francisco, CA, pp. 1-5, 2011.

[7] J. Segovia, M. Cremonesi, C. Cassella, A. Frangi, and G. Piazza, "Experimental Study on the Impact of Anchors Losses on the Quality Factor of Contour Mode AIN Resonators", in proc. 17th International Conference on Solid-State Sensors, Actuators and Microsystems (Transducers 2013), Barcelona, Spain, June 16-20, pp. 2473-2476, 2013.

[8] Y. Hui, and M. Rinaldi, "Aluminum Nitride Nano-Plate Resonant Infrared Sensor with Self-Sustained CMOS Oscillator for Nano-Watts Range Power Detection", in proc. 2013 IEEE International Frequency Control Symposium (IFCS 2013), Prague, Czech Republic, July 21-25, pp. 62-65, 2013.

[9] M. Rinaldi, C. Zuo, J. Van der Spiegel, and G. Piazza, "Reconfigurable CMOS Oscillator based on Multi-Frequency AlN Contour-Mode MEMS Resonators", IEEE Transactions on Electron Devices, vol. 58, pp. 1281-1286, 2011.

[10] M. Rinaldi, C. Zuniga, B. Duick, and G. Piazza, "Use of a Single Multiplexed CMOS Oscillator As Direct Frequency Read-Out for an Array of Eight AlN Contour-Mode NEMS Resonant Sensors", in proc. IEEE Sensors Conference 2010 , Waikoloa, Hawaii, November 1-4, pp. 2666-2670, 2010.

[11] M. Rinaldi, C. Zuniga, C. Zuo, and G. Piazza, "Super High Frequency Two-Port AlN Contour-Mode Resonators for RF Applications", IEEE Transactions on Ultrasonics, Ferroelectrics, and Frequency Control, vol. 57, pp. 38-45, 2010.

[12] M. Rinaldi, Y. Hui, C. Zuniga, A. Tazzoli, and G. Piazza, "High Frequency AlN MEMS Resonators with Integrated Nano Hot Plate for Temperature Controlled Operation", in proc. IEEE International Frequency Control Symposium (IFCS 2012), Baltimore, MD, May 21-24, pp. 1-5, 2012.

[13] D. W. Allan, "Statistics of atomic frequency standards", Proceedings of the IEEE, vol. 54, pp. 221-230, 1966.

[14] http://www.fuji-piezo.com/Pyro1.htm

\section{CONTACT}

*Y. Hui, tel: +1-617-758-9518; yhui@coe.neu.edu 